\title{
Thrombi in antrum atrii dextri of human heart as clinically important source for chronic microembolisation to lungs
}

\author{
THOMAS N JAMES \\ From the Callaway Laboratory of the Department of Medicine, University of Alabama Medical Center, Birmingham, \\ Alabama, USA
}

SUMMARY In many cases of chronic microembolic pulmonary hypertension the source of the emboli is unknown. Disease or injury to the sinus node is usually accompanied by thrombus formation within the antrum atrii dextri, and this region is not often inspected carefully during necropsy. In the present study the hearts of 14 patients with right ventricular hypertrophy or documented pulmonary hypertension showed disease or injury in the sinus node, and each had both old and recent thrombosis in the antrum atrii dextri. In all 14 cases both lungs were available for histological examination. The group included six cases of rheumatic heart disease with mitral stenosis, four cases of disseminated lupus erythematosus, and four cases of so-called primary pulmonary hypertension. Every lung contained both new and old microemboli, as well as a wide variety of other histological abnormalities narrowing the small pulmonary arteries. Many of these narrowing lesions were recognisable as the consequence of prior microembolisation. For future postmortem examination of cases of chronic microembolic pulmonary hypertension, mural thrombi within the antrum atrii dextri should be considered as one important potential source for recurring microembolisation to the lungs.

As a part of many types of cardiac disease, damage to atrial myocardium can be shown almost as often as it is carefully sought. Söderström ${ }^{1}$ reported a masterly review of the subject of atrial infarction and emphasised two points: first, that infarction of the human right atrium was not uncommon and usually included the sinus node; second, that all such infarcts were associated with mural thrombosis, particularly in the antrum atrii dextri, which consists of the convoluted recesses lying between the trabeculae of the free wall of the right atrium and their junctions with the crista terminalis, very close to the sinus node. Two logical corollaries to Söderström's observations are a high incidence of associated atrial arrhythmias, which he emphasised, ${ }^{1}$ and the possible significance of recurring microembolisation to the lung, which was not a subject of his study.

Atrial arrhythmias have long been known to be associated with atrial mural thrombosis, especially if

This work was supported by the National Heart, Lung and Blood Institute and by the Rast Fund for Medical Research

Accepted for publication 21 October 1982 atrial myocardial or endocardial disease coexists or if atrial emptying is impeded as by mitral stenosis. Atrial arrhythmias are perhaps the surest clinical clue to atrial infarction, for example in association with ventricular myocardial infarction..$^{2-4}$ Unless there is chronic insufficiency of coronary blood supply, however, the atrial arrhythmias associated with myocardial infarction would be expected to stop and the mural thrombosis to become either resorbed or effectively covered with endothelium and sealed. On the other hand, there are certain other causes of atrial injury leading to arrhythmias and to mural thrombosis which are not transient but instead are long lasting or else continually recur. Examples include not only the familiar rheumatic heart disease, but also disseminated lupus erythematosus, ${ }^{5}$ polyarteritis nodosa, ${ }^{6}$ Friedreich's ataxia with cardiomyopathy, and so-called primary pulmonary hypertension. ${ }^{8}$

The present study was undertaken to determine how often pulmonary microemboli were demonstrable at necropsy in 14 patients found to have thrombosis in the antrum atrii dextri caused by chronic disease of the sinus node. All 14 patients had 
either clinical evidence (cardiac catheterisation, physical examination) or postmortem findings (right ventricular hypertrophy) indicative of chronic pulmonary hypertension. Clinical diagnoses were rheumatic mitral stenosis (six cases), disseminated lupus erythematosus (four cases), and primary pulmonary hypertension (four cases). One essential criterion for selection of the necropsy cases as described above was the availability of both lungs for histological examination.

\section{Subjects}

Chronic pulmonary hypertension is an important clinical problem in Rheumatic mitral stenosis. Six cases with this diagnosis were studied. None of these patients had received cardiac surgery. There were four women and two men, and their ages ranged from 43 to 75 years. All died of chronic congestive heart failure with severe pulmonary hypertension. All had chronic atrial fibrillation, and each of the six had thrombi in the antrum atrii dextri.

Severe pulmonary hypertension (with microvascular disease) is an infrequent but devastating complication of some cases of disseminated lupus erythematosus. ${ }^{9}$ For the present study the lungs were examined from four cases of lupus in whom mural thrombi were present in the antrum atrii dextri. Three were women and one a man, with ages of 29 to 49 years.

As a third group, the lungs of four patients dying with primary pulmonary hypertension were examined specifically to search for pulmonary microemboli. Three (all women) of these four cases have been the subject of a previous report ${ }^{8}$ dealing principally with abnormalities found in their cardiac conduction system; their ages were 29,31 , and 35 years. The fourth case was a man 28 years old. Criteria for the diagnosis have been presented in detail ${ }^{8}$ but briefly included documented pronounced pulmonary hypertension in the absence of demonstrable causes such as intracardiac shunting, valvular stenosis, or clinical or postmortem evidence for venous thrombosis. In each of the four cases there was a variety of abnormal histological processes which caused narrowing of small coronary arteries (and consequently, focal myocardial degeneration). In all four hearts there was both old and recent thrombosis in the antrum atrii dextri adjacent to chronic disease in the sinus node.

To examine the sinus node appropriately the histological sections necessarily include a comprehensive survey of the entire antrum atrii dextri, ${ }^{1}$ simply because of its anatomical proximity (Fig. 1). Because the anterior margin of the antrum atrii dextri is directly at the junction of the right atrium with its appendage, mural thrombi in the antrum atrii dextri are sometimes mistakenly interpreted to be in the appendage. Methods for this type of histological study have been reported previously. ${ }^{1011} \mathrm{In}$ all of the present hearts one or more samples of myocardium from all four cardiac chambers were also examined. For the pulmonary histological examinations, one or more representative blocks of tissue were excised from each of the five lobes of the two lungs; the size of each excised block permitted sections measuring no less than 2 by $5 \mathrm{~cm}$ in area. Multiple sections were cut from each block of pulmonary tissue. All sections were $8 \mu$ thick. From every block some slides were prepared with both Goldner trichome and Verhoeffvan Gieson elastic stains. Selected sections were stained with the periodic acid Schiff method.

\section{Pathological findings}

\section{GENERAL FINDINGS}

In all 14 hearts there was focal damage within the sinus node, some recent (haemorrhage, infarction, oedema, inflammation) and some old (abnormal fibrosis, fatty replacement). Except in the antrum atrii

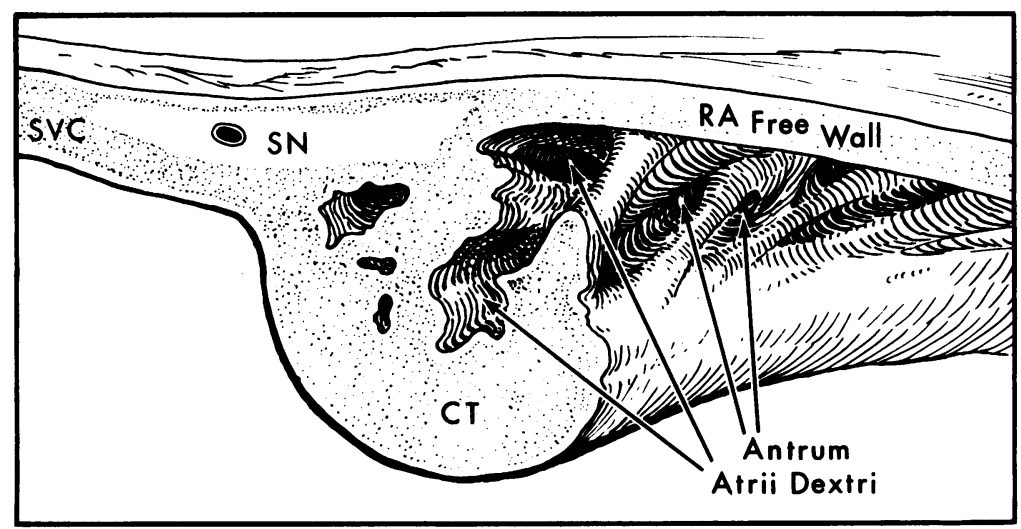

Fig. 1 This drawing illustrates the location of the antrum atrii dextri of the human heart, and its anatomical relation to the sinus node $(S N)$, crista terminalis $(C T)$, and right atrial (RA) free wall. The cut depicted extends from superior vena cava $(S V C)$ through the $S N$, perpendicular to the line of the CT. 
dextri, there were no other mural thrombi in the right atrium or its appendage. The right ventricle was hypertrophied in all 14 hearts. A few scattered small mural thrombi were found in the right ventricles of three of the 14 hearts, two being from the six patients with rheumatic mitral stenosis and the third from a patient with lupus erythematosus. There were no thrombi on and there was no intrinsic disease of the tricuspid or pulmonary valves. No peripheral venous thrombosis was found in any of these 14 patients; it is recognised, of course, that small peripheral venous thrombi may have been missed.

Both old and recent evidence of mural thrombosis was present in the antrum atrii dextri of every heart. Older thrombi had become organised, some with myxoid elements and others with lamellar fibrosis layered within recesses of intertrabecular crevices. Newer thrombi were adherent over varying lengths of endocardium, some being attached only tenuously and appearing to be readily dislodged. Fresh thrombi sometimes attached over old organised thrombi as well as directly to endocardium. On gross examination the older thrombi had the appearance of small lima beans when seen from the endocardial surface of the right atrium.

Small pulmonary arteries were focally narrowed by a variety of histological processes in all 14 cases. In every lung both old and more recent emboli were present in the small pulmonary arteries, most being 1 $\mathrm{mm}$ or less in diameter. No effort was made to quantify the number of pulmonary microemboli; they were present and easily found, however, in every histological section of lung in all 14 cases. Study of 10 or more serial sections (total distance in any series comprising about $100 \mu$ ) from representative blocks of pulmonary tissue of each of the 14 cases amply showed the focal nature of the vascular disease, the same pulmonary artery being variably narrowed or widely patent at different points. Serial sections also helped establish whether a thrombus was antemortem in nature. Though a number of different abnormalities caused narrowing of the small pulmonary arteries, the spectrum of arterial changes was consistent within any given disease group.

\section{RHEUMATIC MITRAL STENOSIS}

The variety of pulmonary arterial lesions found in the six cases of rheumatic heart disease was characterised primarily by a much thickened tunica media of most pulmonary arteries of any size. In some of these there was fibrosis of the medial layer of the pulmonary arteries and in others there was a variable amount of intimal proliferation. There was no associated inflammation within or around these pulmonary arteries. Examples of degeneration in the sinus node, clots in the antrum atrii dextri, and the embolic pul- monary vascular disease from one patient with $c$ rheumatic heart disease (Fig. 2-4) are characteristic of $\vec{F}$ the findings in all six patients with this diagnosis.

\section{DISSEMINATED LUPUS ERYTHEMATOSUS}

Of the four cases of disseminated lupus $\frac{\bar{D}}{\bar{D}}$ erythematosus, there was scattered arteritis present in $\triangle$ the lungs of one but no pulmonary arteritis in the other three. All four cases exhibited intimal prolifera- $\overrightarrow{0}$ tion, old and recent microemboli, and varying degrees of thickening of the tunica media in the small pulmo- $\vec{\omega}$ nary arteries. In no case was the medial hypertrophy as extensive as in the patients with rheumatic mitral stenosis. Examples from the sinus node and the lungs $\overrightarrow{0}$ of one of these four patients (Fig. 5 and 6) are charac- ? teristic of the group.

\section{PRIMARY PULMONARY HYPERTENSION}

Findings in all four cases of primary pulmonary $\vec{T}$ hypertension were similar, clinical features of three of $\mathbb{D}$ these having been reported previously. ${ }^{8}$ In all four cases there were narrowing abnormalities not only as $\frac{0}{2}$ expected in the pulmonary arteries but also in many $\vec{\imath}$ small coronary arteries. The coronary lesions were $\stackrel{\infty}{\infty}$ associated with focal necrosis and both recent and chronic focal degeneration within and near the sinus node, associated with the formation of extensive old and recent mural thrombosis within the antrum antri dextri (Fig. 7). Microemboli were present in the small arteries of every lung studied (Fig. 8). There was a $\stackrel{\varnothing}{\circ}$ conspicuous absence of medial hypertrophy in the $\overrightarrow{\vec{O}}$ pulmonary arteries, and there was medial hypop- $\frac{3}{3}$ lasia. 1213 The thin tunica media (Fig. 8) showed little scarring or fibrosis, but there was rarely some focal medial degeneration, similar to that seen in the small coronary arteries. A radial pattern of intimal proliferation 1415 frequently caused distinct narrowing of the pulmonary arterial lumen (Fig. 8B). Scattered 3 "angiomatoid" lesions were present in the lungs of all four cases. There was no evidence of inflammation $\frac{3}{3}$ within or near any of the pulmonary or coronary arteries.

\section{Discussion}

For most physicians embolism from the left side of $N$ the heart is a more familiar medical problem than $N$ emboli originating from the right side. This is under- $\omega$ standable in part because even small emboli lodging in $<$ the brain or the kidney, for example, have $\operatorname{such}^{\circ}$ dramatic and easily recognised clinical manifestations. Small pulmonary emboli, on the other hand, can and ${ }_{T}^{+}$ usually do escape clinical recognition unless they con- $-\frac{0}{0}$ tinue to recur for a long time. That recurrent pulmo- $\overrightarrow{\mathbb{D}}$ nary microemboli can lead to serious pulmonary $\frac{?}{0}$ hypertension has been reported in association with $\frac{0}{0}$ 


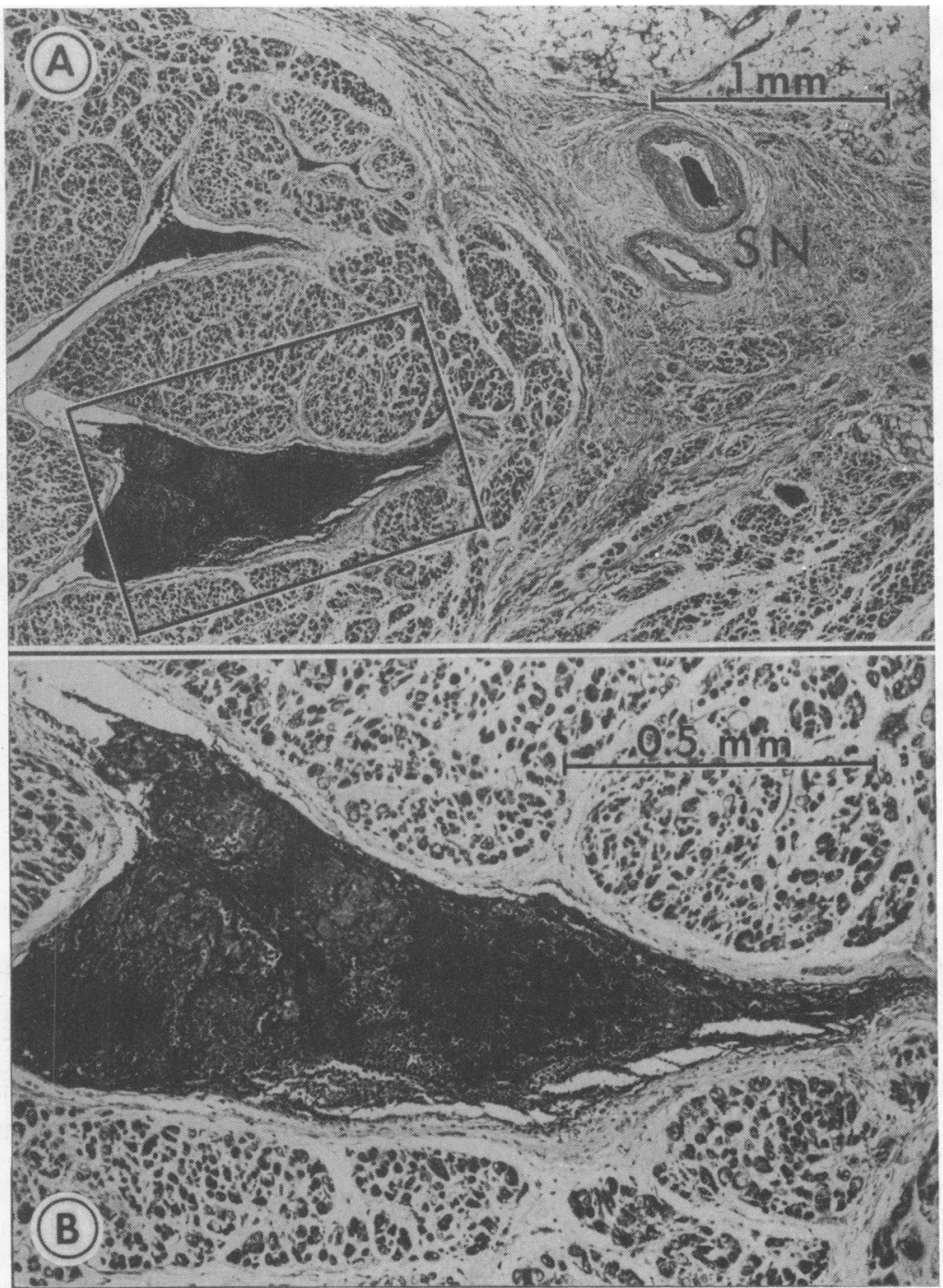

Fig. 2 This and Fig. 3 are photomicrographs of the sinus node and thrombus-filled antrum atrii dextri from one of the patients with rheumatic heart disease. Boxed area in $(A)$ encompasses a mural thrombus, seen at higher magnification in $(B)$. In every photomicrograph magnification is indicated with a reference bar. Goldner trichrome stain here and in every photomicrograph unless otherwise indicated.

tumour embolisation to the lung 1617 and with emboli originating within portocaval shunts. ${ }^{18}$ Despite the general recognition, however, that chronic recurring microemboli can be responsible for chronic pulmonary hypertension, experienced investigators have emphasised that for most cases the source of such emboli has remained unknown. 1920

Whether chronic microemboli to the lungs can be a sufficient cause for sustained pulmonary hypertension if the pulmonary arteries are not already abnormal is uncertain. It is likely, for example, that occasional showers of such small thromboemboli can be readily dissolved or resorbed within the normal pulmonary circulation. For patients in whom microemboli originate in the right side of the heart, however, there are at least two reasons to expect that they may already have an abnormal pulmonary circulation. The first reason is the frequent coexistence of coronary and pulmonary arterial abnormalities (both probably primary) in many diseases. ${ }^{5-8}$ The second reason is the frequent presence of secondary pulmonary hypertension (before chronic microembolisation to the 




Fig. 3 Focal degeneration within the sinus node is shown at higher magnification from the same heart seen in Fig. 2 (rheumatic heart disease).

lungs) when there is some chronic myocardial disease leading to left ventricular failure, or when there is some other cause of impaired pulmonary venous return to the heart as in mitral stenosis.

In as disparate a group of diseases as rheumatic mitral stenosis, disseminated lupus erythematosus, and primary pulmonary hypertension, the present study shows that there are clearly some cases with thrombosis in the antrum atrii dextri associated with microembolisation into the lungs. All 14 of the cases chosen for the present study had this in common. How often, however, clots in the antrum atrii dextri may be present among all patients dying with, for example, rheumatic mitral stenosis, will require a different study from the present one. The purpose here was to see how often pulmonary microemboli were present in patients who were found at necropsy to have thrombosis in the antrum atrii dextri. The answer in these 14 selected cases was that in all of them pulmonary microemboli were abundantly present and must have contributed to either the pathogenesis or the worsening of the pulmonary vascular disease.

How large a role pulmonary microembolisation played in the development or progression of pulmonary hypertension must have varied greatly in these three different diseases. But certain things can be deduced to be present in all three diseases and all 14 cases. Since every heart contained both old and recent thrombi within the antrum atrii dextri, it seems highly probable that showers of microemboli were a recurring problem in all of them. Because of the small size of individual emboli, it is unlikely that any one of them accounted for clinically recognisable symptoms. One may further question whether most episodes could have been detected in vivo even with more sophisticated examinations such as isotopic ventilation-perfusion scans. On the other hand, it is known from experimental studies that clot fragments and debris can act as powerful stimuli for intimal proliferation ${ }^{2122}$ with inevitable narrowing of arterial lumens. Thus, in addition to the initial mechanical obstruction produced by multiple microemboli in scattered small pulmonary arteries, perhaps only temporarily in those vessels where active fibrinolysis was effective, there is the more sustained effect occurring as a consequence of the induced intimal proliferative process. Furthermore, once the endothelium is so altered, it may be less capable of fibrinolytic dissolution $^{23}$ of any subsequent small emboli which may randomly lodge there.

Another type of transient consequence may occur secondary to the release of serotonin during the process of repeated or continuing mural thrombosis within the antrum atrii dextri. The release of seroto- 


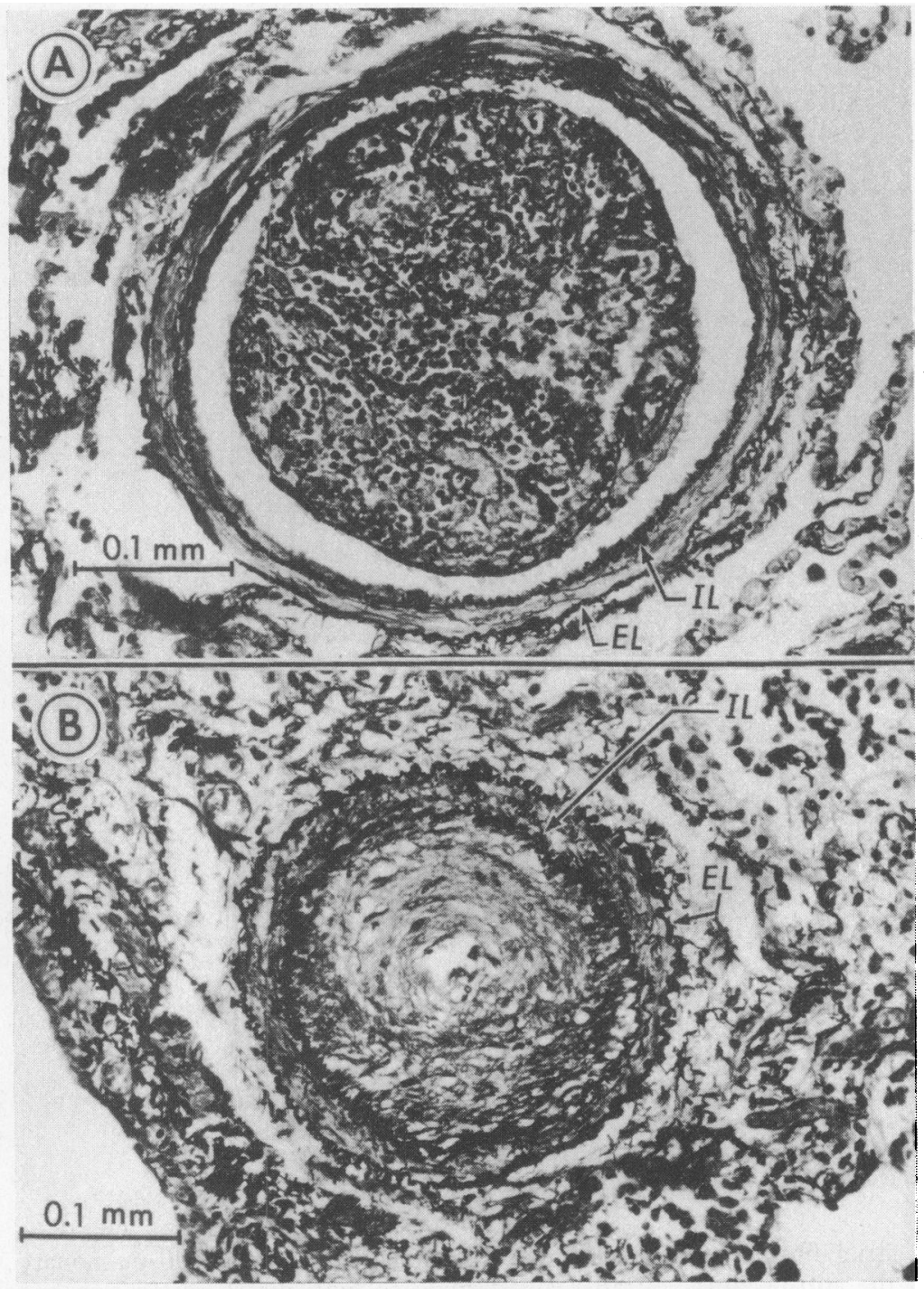

Fig. 4 Representative examples of pulmonary arterial disease found in these patients with rheumatic heart disease are shown here (same patient as Fig. 2 and 3). Embolus seen in (A) distends and thins the wall of the pulmonary artery. An old mixture of lamellar intimal hyperplasia, fragmentation of the elastic lamina, and other thickening processes virtually obliterate the lumen of the pulmonary artery in $(B)$. The tunica media is seen between the internal and external elastic laminae, marked IL and $E L$, respectively. Verhoeff-van Gieson elastic stain in $(A)$ and $(B)$.

nin (and other vasoactive substances) from platelets would only be expected to occur during the initial steps in the process of thrombosis. After its release, serotonin arriving within the pulmonary circulation would meet with rapid metabolic degradation, but it should be remembered that the thrombus formation was probably a continuing or recurring process. Furthermore, as some of the pulmonary arteries became structurally abnormal, particularly their endothelium, the expeditious local destruction of serotonin there could become impaired. Since the familiar and powerful constricting effect of serotonin on the pulmonary circulation must have been repeatedly introduced, even this transient humoral influence could become recurrently significant.

Returning to the question of the exact nature of the functional importance of recurring microembolisation from the right atrium, it probably differed for each disease. In the chronic pulmonary hypertension of rheumatic mitral stenosis, for example, the small pulmonary arteries had already become narrowed by reactive medial hypertrophy, some smooth muscle later being variably replaced by fibrosis. Recurring microemboli from the antrum atrii dextri to the small pulmonary arteries must seriously compound the pulmonary hypertension already present because of 
mitral valve obstruction. Chronic atrial fibrillation would further facilitate clotting in the antrum atrii dextri.

In disseminated lupus erythematosus clinically important pulmonary hypertension does occur ${ }^{9}$ but it is rare. Such cases have sometimes had pulmonary arteritis. ${ }^{24}$ Other clinical manifestations of disseminated lupus usually are much more important, such as the well known ones in the kidneys, the brain, or the heart. In the four cases of the present study the principal value is the illustration of an association between clots in the antrum atrii dextri and the narrowing abnormalities in the pulmonary arteries.

Finally, in those patients with so-called primary pulmonary hypertension the same compounding cycle of events must be suspected: insidious thrombi in the antrum atrii dextri forming silently but recurrently, dislodging to embolise into the small pulmonary arteries and leading to both direct obstruction and 을 proliferative stimulation of pulmonary arterial intimal $\rightarrow$ cells. Chronic pulmonary embolisation is usually con- 을. sidered to be an important differential diagnosis in $\bar{N}$ such patients, but the source of such emboli has sel- $\sigma$ dom been identified or even suggested. With the $\tilde{O}$ demonstration of such a source in the antrum atrii $\underset{\omega}{N}$ dextri, further attention must be directed at this type 0 of recurring microembolisation. It would not explain 6

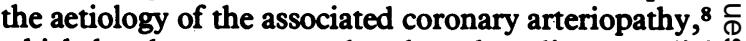
which has been suspected to be a nereditary medial $\stackrel{\infty}{\rightarrow}$ necrosis. But the coronary arteriopathy causes focal 0 myocardial degeneration which leads to recurring $\stackrel{\vec{P}}{\vec{P}}$ mural thrombosis in the right atrium, then to chronic $\stackrel{\mathbb{D}}{\circ}$ microembolisation and, eventually, permanent pul- $\stackrel{\mathbb{Q}}{\Omega}$ monary arterial narrowing. 


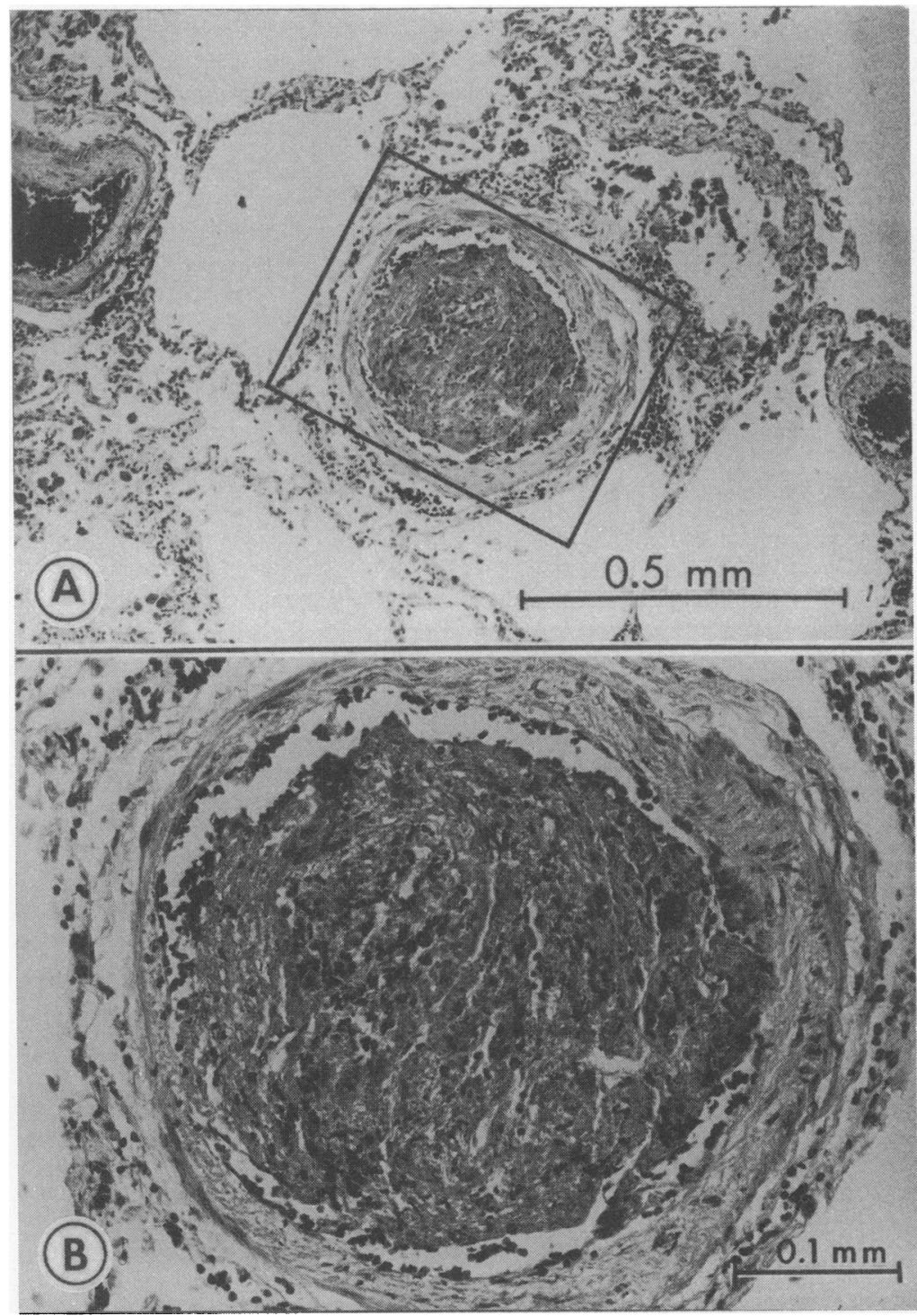

Fig. 6 Microembolism in a small pulmonary artery of the case in Fig. 5 is shown here at two magnifications.

No claim is made that all microembolic pulmonary hypertension originates in the antrum atrii dextri, for the study was not designed to determine what percentage does. What can be said is that in six cases of rheumatic mitral stenosis, four of lupus erythematosus, and four of primary pulmonary hypertension, all of whom had thrombi in the atrum atrii dextri, each had both old and recent pulmonary microemboli. For many patients dying with microembolic pulmonary hypertension the source of such emboli has been emphasised to be obscure or unknown. ${ }^{19} 20$ Careful examination of the antrum atrii dextri is clearly merited in all future postmortem studies of such deaths. 


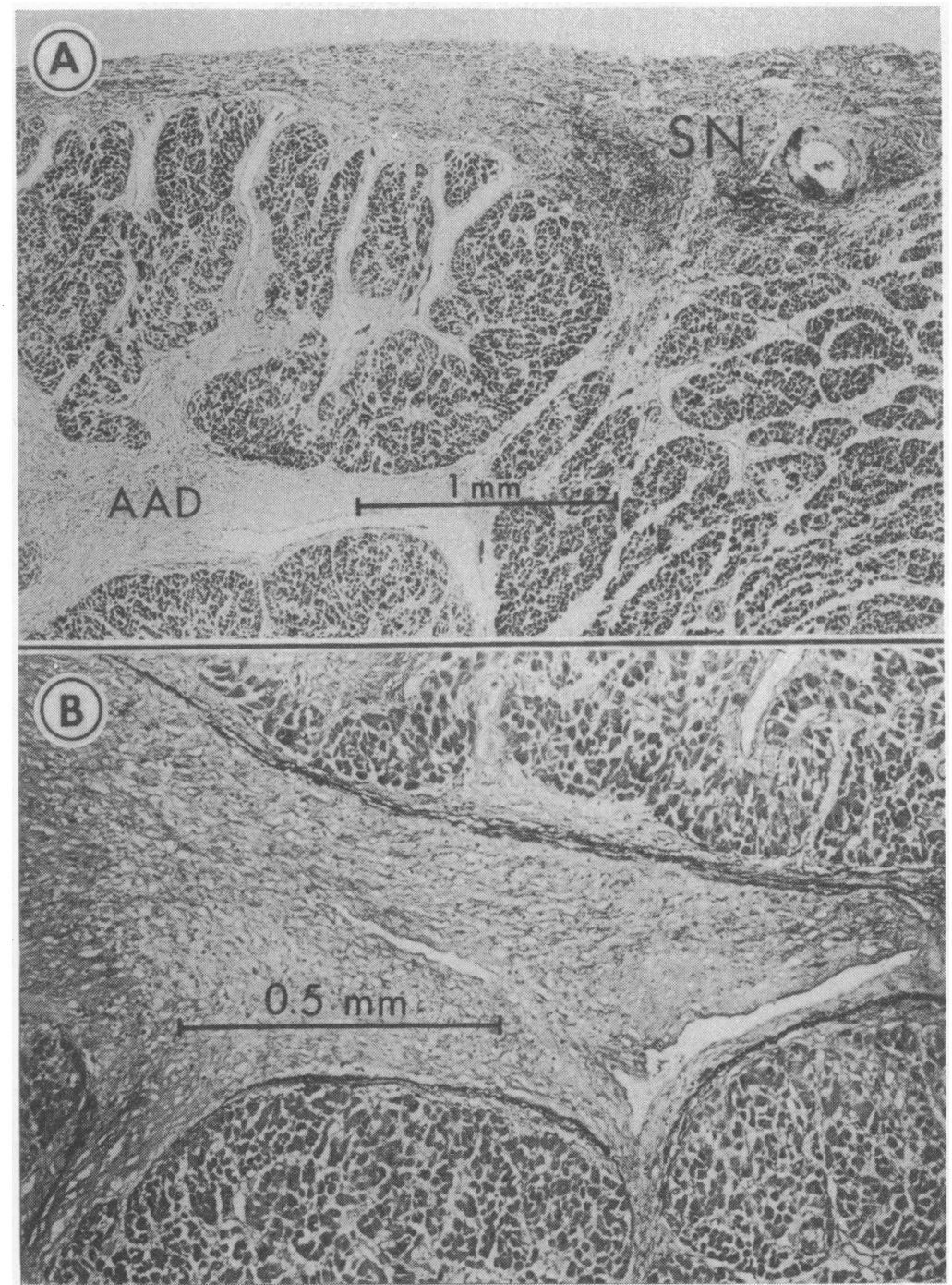

Fig. 7 The region of the sinus node and antrum atrii dextri are shown here from the heart of a patient dying with primary pulmonary hypertension. There is degeneration and haemorrhage in the sinus node $(S N$ in $(A))$, and an old organised mural thrombus in the adjacent atrum atrii dextri $(A A D$, seen in $(A)$ and $(B))$. Elastic stain in $(B)$. Two typical pulmonary arteries from this same patient are shown in Fig. 8.

\section{References}

1 Söderström N. Myocardial infarction and mural thrombosis in the atria of the heart. Acta Med Scand 1948; suppl 217: 7-114.

2 Cushing EH, Feil HS, Stanton EJ, Wartman WB. Infarction of the cardiac auricles (atria): clinical, pathological, and experimental studies. Br Heart $\mathcal{F} 1942$; 4: 17-34.

3 James TN. Myocardial infarction and atrial arrhythmias. Circulation 1961; 24: 761-76.
4 James TN. The coronary circulation and conduction system in acute myocardial infarction. Prog Cardiovasc Dis 1968; 10: 410-49.

5 James TN, Rupe CE, Monto RW. Pathology of the cardiac conduction system in systemic lupus erythematosus. Ann Intern Med 1965; 63: 402-10.

6 James TN, Birk RE. Pathology of the cardiac conduction system in polyarteritis nodosa. Arch Intern Med 1966; 117: $561-7$.

7 James TN, Fisch C. Observations on the cardiovascular involvement in Friedreich's ataxia. Am Heart $\mathcal{F}$ 1963; 66: 


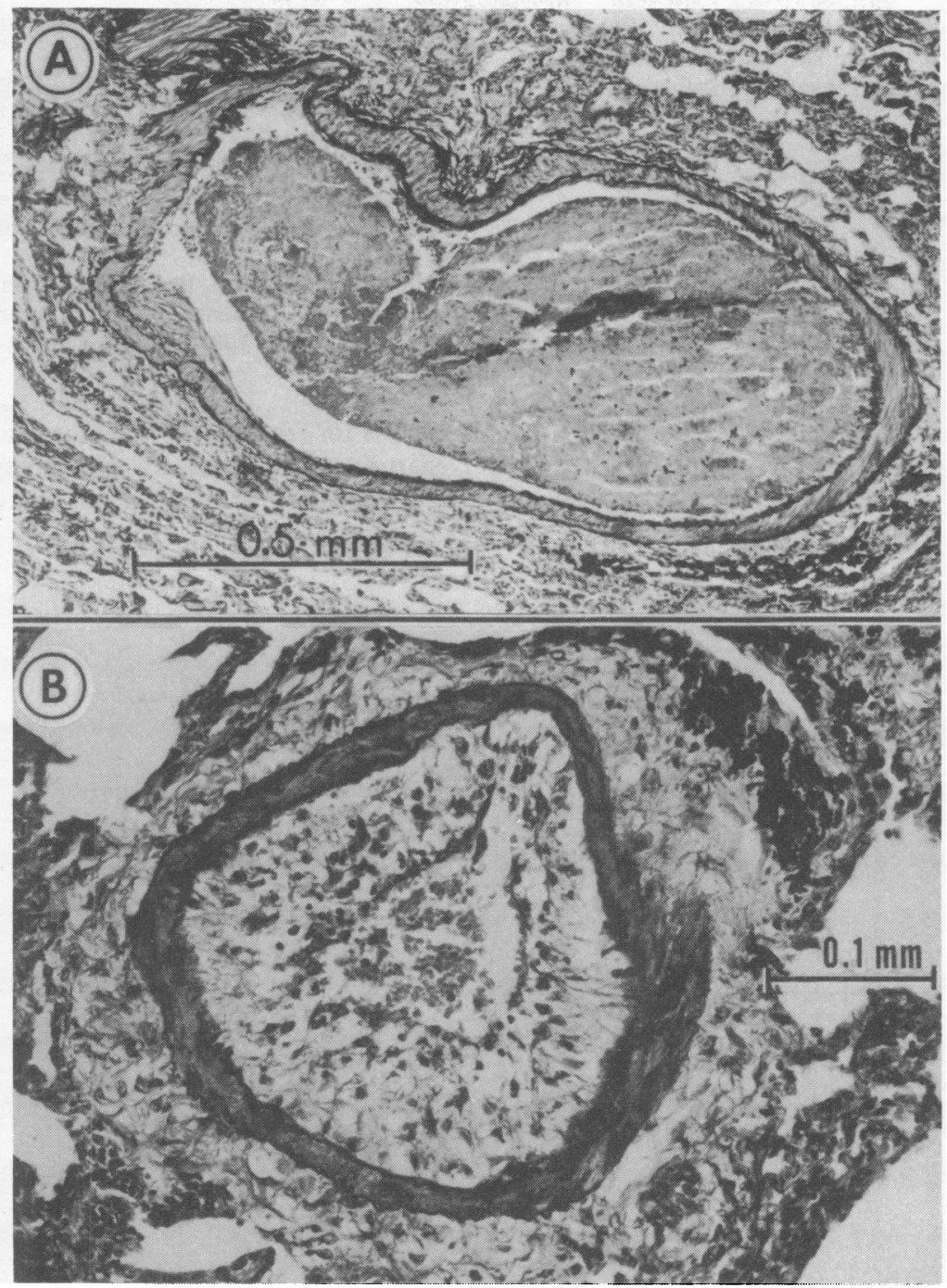

Fig. 8 Microembolism in a pulmonary artery is shown in (A). A radial pattern of intimal proliferation and thin tunica media are seen in $(B)$. The tunica media is seen clearly between the internal and external elastic laminae stained black in each artery (Verhoeff-van Gieson stain).

$164-75$.

8 James TN. On the cause of syncope and sudden death in primary pulmonary hypertension. Ann Intern Med 1962; 56: $252-64$.

9 Sack KE, Bekheit S, Fadem SZ, Bedrossian CWM. Severe pulmonary vascular disease in systemic lupus erythematosus. South Med F 1979; 72: 1016-8.

10 James TN. Anatomy of the human sinus node. Anat Rec 1961; 141: 109-39.

11 James TN. The sinus node. Am $\mathcal{F}$ Cardiol 1977; 40: 965-86.

12 Bredt H. Die primäre Erkrankung der
Lungenschlagader in ihren verschiedenen Formen. Virchows Arch Pathol Anat 1932; 284: 126-53.

13 Gilmore JR, Evans W. Primary pulmonary hypertension. F Pathol Bacteriol 1946; 58: 687-97.

14 Brenner O. Pathology of the vessels of the pulmonary circulation (part I). Arch Intern Med 1935; 56: 211-37.

15 Brenner O. Pathology of the vessels of the pulmonary circulation (part III). Arch Intern Med 1935; 56: 725-52.

16 Feller HA, Janis JF. Pulmonary hypertension, resulting from tumour emboli to pulmonary arteries. Dis Chest 1968; 54: 68-70. 
17 Brisbane JU, Howell DA, Bonkowsky HL. Pulmonary hypertension as a presentation of hepatocarcinoma. Report of a case and brief review of the literature. $A m \mathcal{F}$ Med 1980; 68: 466-9.

18 Sallam M, Watson WC. Pulmonary hypertension due to micro-thromboembolism from splenic and portal veins after portacaval anastomosis. Br Heart $\mathcal{F}$ 1970; 32: 269 71.

19 Mlczoch J, Tucker A, Weir EK, Reeves JT, Grover RF. Platelet-mediated pulmonary hypertension and hypoxia during pulmonary microembolism. Chest 1978; 74: 648-53.

20 Fowler NO, Black-Schaffer B, Scott RC, Gueron M. Idiopathic and thromboembolic pulmonary hypertension. Am $\mathcal{F}$ Med 1966; 40: 331-45.

21 Wartman WB, Jennings RB, Hudson B. Experimental arterial disease. I. The reaction of the pulmonary artery to minute emboli of blood clot. Circulation 1951 ; 4: 747_C. 55.

22 Geer JC, Glass A, Albert HM. Pulmonary vascular lesions in the dog produced by autogenous clot embol은 ism. Exp Mol Pathol 1965; 4: 391-8.

23 Metcalfe DD, Lewis RA, Silbert JE, Rosenberg RD, Wasserman SI, Austen KF. Isolation and characteriza- 8 tion of heparin from human lung. $\mathcal{F}$ Clin Invest 1979; 64:0 1537-43.

24 Nair SS, Askari AD, Popelka CG, Kleinerman JF. Pul- $\vec{\circ}$ monary hypertension and systemic lupus erythematosus. Arch Intern Med 1980; 140: 109-11.

Requests for reprints to Professor Thomas N James $\frac{1}{50}$ Department of Medicine, University of Alabama Medical Center, Birmingham, Alabama 35294, USA. 